\title{
ELECTRICITY IN PLANTS.
}

\author{
BY Edwin Smith, M. A.
}

THERE is a very interesting chapter in Becquerel's smaller treatise (in three volumes) on "Ilectricity and Magnetism " concerning the electrical phenomena of plants. I was induced, by reading this chapter, to make a series of experiments, with a view to illustrate the author's statements, and, if possible, to throw some fresh light upon an obscure and puzzling subject. I am now going to put together some notes of these experiments, carefully made at the time, in the hope that they may not be without a certain interest to others. The galvanometer employed is a home-made one, but tolerably sensitive. My greatest difficulty has been to depolarise the slips of platinum by means of which I have made the voltaic circuits; but every care has been taken, to the best of my ability, to obviate error arising from this cause, as well as from thermo-electric disturbance.

Experiment 1.-Take the thick, juicy leaf stalk of the common garden rhubarb, and, having cut off a length of from 4 to 18 inches, apply a square of platinum foil to each end of the piece. Connect by means of platinum wire, these two plates with opposite terminals of the galvanometer. The needle will be powerfully deflected. Now reverse the position of the rhubarb between its platinum surfaces, connect as before, and the needle will again be deflected, but in an opposite direction. Having previously ascertained how to interpret the movements of the needle, by noticing its behaviour when a voltaic current is produced between zinc and diluted acid, with platinum for negative plate, and transmitted through the coil, you will at once conclude that a current of electricity is passing from that end of the leaf stalk nearcst the root towards the opposite end, nearest the blade of the leaf.

Experiment 2,-Cut off a piece about 3 inches long from the thickest part of the same leaf-stalk. Divide it longitudinally down the middle. Pare off a level slice of the outer cuticle. Lay the platinum slips close to the two flat sides thus provided, and connect with the galvanometer. There will be indicated a current from the outer side nearest the cuticle to the inner axis of the leaf-stalk. If the section is made thinner, there will be a current in the same direction. You may confirm your conclusion, as before, by simply turning over the piece of rhubarb, so as to reverse the current. Experiments 1 and 2 may be tried upon a thick midrib or branch of the same, taken from the leaf-blade, with a precisely similar result. A cucumber acts in the same way.

Experiment 3.--Insert a platinum wire into the lower end of the flower-stalk of peony. Apply foil to one of the large bracts, or to a petal; connect, ard observe deflection. A current is detected flowing from the lower end of the flower-stalk to the bract or petal. 'the same experiment may be tried with a sprig of fresh oak-leaves, or bramble, or chickweed, or bunch of elder-buds, or poppy-flower, 
or stalk and leaves of poppy. The current always sets from the point nearest to the root towards the organs furthest from the root. If wires be inserted in the pistil of a flower and its stalk, there will be shown a current flowing towards the former.

Experiment 4.-Lay a leaf of the common dock between pieces of platinum foil. Connect the different surfaces with opposite terminals of the galvanometer, and notice the deflection. A current is indicated from the upper to the under surface of the leaf. A leaf of Spanish chestnut or of young elder will give the same result; but in four experiments on a leaf of sycamore, I got a current from the under to the upper surface, after a momentary current in an opposite direction. I infer, however, that the momentary current was the true one, and that the second arose from polarisation; for I obtained this latter current by merely employing filter-paper dipped in distilled water, and laid between the slips of foil.

Experiment 5.--'Twist a piece of wire round the outer cuticle of a young twig of hawthorn. Insert a second wire between the bark and wood, so as to touch the layer of cambium. On completing the circuit, a current will be indicated from the cambium to the outer cuticle. Again : pass a wire into the pith, and a current may be detected taking a direction from the cambium to the pith. Instead of hawthorn, a cutting from a fresh branch of elder, laurel, hedge-mustard, thistle, groundsel, yarrow or other dicotyledonous plant, may be employed, with, as I have uniformly found, the same effect.

Experiment 6.-Scrape off the outer cuticle of the root of cress. By means of wires, as before, test the direction of the electric cur. rents. They will be found to flow from the outside to the axis of the root, and again, from the root-stock, or point of junction with the stem, towards the lower tapering end. The same effects are exhibited by the root of cat's ear, or of thistle. If plates of platinum foil are applied to the flat surfaces made by cutting across the thick and tbin ends of a carrot, a current will be found to set from the thick end of this root to the smaller end.

Experiment 7.- Take a bunch of the seeds of sycamore. Dip a sumara attached to the stalk near its broader end into one of the mercury cups, and a samara attached to the stalls at a point nearer the smaller end into the other cup. A current is indicated from the former towards the latter, in strict analogy to what happened in the third experiment. Or a piece of the stalk itself may be bent, and its ends dipped into the mercury-cups. A current will be indicated, having the same direction as before. A cutting from any pliable stem may be tried in this way, and always, so far as my experience goes, with a similar result.

Experiment 8.-Pass a platinum wire through the tubular hol. low of a stem of Poa, or some other meadow grass. Twist another platinum wire rouud the outside, and connect both with the galvanometer. A current will be shown, having a direction from the 
interior to the exterior surface of the stem in question. Such appears to be the law with the stems of monocotyledonous plants.

Experiment 9.-Make two cuts through a raw potato, one through the centre, another near the outer coat. Apply strips of platinum to the two flat surfaces, and connect with opposite terminals of the galvanometer. A current is shown to be passing from the centre towards the outside. A lemon, treated in the same way, furnished a current from the outside towards the centre. With a turnip, a gooseberry, and a pear, the same result was obtained; but a stalk of asparagus followed the law of the potato. A boiled potato, tested, when cold, by the same method, gave a current from the outside towards the centre, the indications being confirmed by a reversal of the slice between the platinum conductors. But, after moistening a fresh piece of boiled potato with distilled water, a current was obtained from the centre towards the outside-a result confirmed by reversing the position of the slice between the platinum conductors, as in the previous trial. Fresh platinum was employed, to obviate, as far as possible, any error from polarisation.

Experiment 10.-Reduce to a pulp, with distilled water, some of the meal nearest the coat of a baked potato. Then make a simi. lar pulp of some of the meal at the centre. Having placed a plug of tow at the bend of a U-tube, pour the pulps thus prepared into different limbs. Connect, by slips and wires of platinum, with the galvanometer. In my experiment, a current was indicated from the outer pulp towards the inner pulp, agreeably to the result of my first experiment with a slice of boiled potato. My object was to destroy all vitality in the tuber by boiling or baking, and to see whether any chemical action might still remain--sufficient to cause a voltaic current when meal from different parts of the potato was made to furnish a voltaic couple.

Experiment 11.--Having procured a garden nasturtium growing in a flower-pot, insert a wire in the fleshy stem, a few inches above the soil. Then thrust a second wire through the small hole at the bottom of the pot into the soil. Connect with galvanometer. The needle is so deflected as to indicate the passing of a current from the plant to the soil in which it grows. Watering the soil made no change in the direction of the current when I performed the experiment. I also stuck the first wire into the stem in various ways and in various parts, always with the same results. At the same time, a current was detected passing from the root end towards the leaf end of the stem, conformably to the rule in other cases.

Experiment 12.--Make a solution of oxygen in distilled water, also a solution of carbonic acid. Pour them into the different limbs of a U-tube furnished with a plug of tow. Insert platinum slips, and connect with galvanometer. There will be a marked deflection of the needle, showing the passage of an electric current from the oxygen to the carbonic acid. Confirm the result by reversing the platinum connectors. 